\title{
Evidence for a global oculomotor program in reading
}

\author{
Noor Al-Zanoon ${ }^{1} \cdot$ Michael Dambacher $^{2} \cdot$ Victor Kuperman $^{3}$
}

\begin{abstract}
Recent corpus studies of eye-movements in reading revealed a substantial increase in saccade amplitudes and fixation durations as the eyes move over the first words of a sentence. This start-up effect suggests a global oculomotor program, which operates on the level of an entire line, in addition to the well-established local programs operating within the visual span. The present study investigates the nature of this global program experimentally and examines whether the start-up effect is predicated on generic visual or specific linguistic characteristics and whether it is mainly reflected in saccade amplitudes, fixation durations or both measures. Eye movements were recorded while 38 participants read (a) normal sentences, (b) sequences of randomly shuffled words and (c) sequences of z-strings. The stimuli were, therefore, similar in their visual features, but varied in the amount of syntactic and lexical information. Further, the stimuli were composed of words or strings that either varied naturally in length (Nonequal condition) or were all restricted to a specific length within a sentence (Equal). The latter condition constrained the variability of saccades and served to dissociate effects of word position in line on saccade amplitudes and fixation durations. A robust start-up effect
\end{abstract}

Noor Al-Zanoon

nalzanoo@western.ca

1 Department of Health and Rehabilitation Sciences, University of Western Ontario, 1151 Richmond St, London, ON N6A 3K7, Canada

2 University of Leicester, Leicester, UK

3 McMaster University, Hamilton, Canada emerged in saccade amplitudes in all Nonequal stimuli, and-in an attenuated form-in Equal sentences. A startup effect in single fixation durations was observed in Nonequal and Equal normal sentences, but not in z-strings. These findings support the notion of a global oculomotor program in reading particularly for the spatial characteristics of motor planning, which rely on visual rather than linguistic information.

\section{Evidence for a global oculomotor program in reading}

Reading requires a precise, rapid and time-locked orchestration of complex oculomotor, visual and cognitive processes. A recent paper by Liversedge, Drieghe, Li, Yan, Bai, and Hyönä (2016) succinctly characterizes the relationship between a system subserving the uptake of visual information and a system responsible for linguistic processing as antagonistic, "with each system having the potential to constrain the other" (p. 66). Not only do eyemovements regulate what parts of a text are being processed at which moment, but also the printed material affects the way the eyes move through a text (Balota, Pollatsek, \& Rayner, 1985; Balota \& Rayner, 1983). The latter influences of the visual input on eye movements are expected, because the oculomotor system is engaged to guide the eyes such that the cognitive system receives an amount of visual and linguistic information that is neither too small nor too large, but rather can be effectively (if not exhaustively) processed between saccades (Just \& Carpenter, 1980). As a result, latencies, durations, amplitudes and landing positions of saccades are contingent, to a degree, on the spatial layout of printed information as well 
as the linguistic complexity (e.g., word frequency, semantics, syntax and punctuation) of read material (Rayner \& Duffy, 1986). Importantly, existing models of oculomotor control in reading vary in how much eyemovements are directed by visual information (Engbert, Nuthmann, Richter, \& Kliegl, 2005; Reichle, Warren, \& McConnell, 2009). The identification of factors that mutually constrain and influence relevant motor, perceptual and cognitive systems presents a validation point for model assumptions and a major challenge for empirical studies. This paper addresses this challenge by carefully decomposing the influence of key linguistic and oculomotor factors.

One proposed implication of the relationship between the oculomotor, visual and cognitive systems is that readers develop an oculomotor program that optimizes the timing and the scope of their uptake of visual information. By far, the most studied type of such an oculomotor program is the one operating within the boundaries of the perceptual span, i.e., at a scale of one to three words (Engbert et al., 2005; Reichle, Rayner, \& Pollatsek, 2003). There have been proposals, however, that readers may plan and launch oculomotor programs that are attuned to maintaining efficient processing on a more global scale, i.e., for an entire line or a paragraph of text. To our knowledge, the first such proposal stemmed from Pynte and Kennedy's (2006) observation that the amplitudes of incoming saccades increase as the eyes move further from left to right in the lines of English and French texts:

[t]he shape of the curves, with shorter saccades at the beginning and end of the line, points to the existence of a motor program modulating saccade planning over a whole line of text at a time (Pynte \& Kennedy, 2006, p. 3790).

Kuperman, Dambacher, Nuthmann, and Kliegl (2010) have further adapted the notion of a global oculomotor program: the planning of eye-movements over an entire line of text. They demonstrated a sharp increase in single fixation and gaze durations as the eyes moved rightwards through the first few words of the sentence (labeled a startup effect), followed by a plateau with relatively stable fixation durations in the middle of the sentence, and another sharp increase in the final words of the sentence (labeled a wrap-up effect, cf. Balogh, Zurif, Prather, Swinney, \& Finkel, 1998; Rayner, Kambe, \& Duffy, 2000; Rayner, Sereno, Morris, Schmauder, \& Clifton, 1989). This nonlinear pattern has been found in eye-tracking data from three sentence-reading experiments in German and Dutch, and in the paragraph-final lines of texts in the Dundee corpus (Kennedy \& Pynte, 2005). Kuperman et al. (2010) have argued that the effect of word position on fixation durations was a compound effect: the wrap-up effect has a linguistic cause (Hill \& Murray, 2000; Hirotani, Frazier, \& Rayner, 2006; Warren, White, \& Reichle, 2009), while the start-up effect has an oculomotor cause. More specifically, Kuperman et al. (2010) have observed that the initial increase in fixation durations comes together with a dramatic increase in saccade amplitudes as the eyes moved through the first few words of a Dutch or German sentence, similarly to the effect reported by Pynte and Kennedy (2006). In contrast to Pynte and Kennedy, though, saccade amplitudes stayed flat or increased slightly on later positions in the sentence, but did not become shorter (Fig. 5 in Kuperman et al., 2010). Yet, the pattern supports the idea of a global oculomotor program that aims at maintaining an efficient and rapid progression through a line.

Several other studies corroborate the possible existence of processing strategies or programs operating over lines or paragraphs. For example, distinct clusters of eye-movement patterns have been observed during reading of expository texts: the symptomatic features of these patterns included the presence or absence of looks-back to previous sentences, the overall speed of reading and the amount of attention paid to the text headings (Hyönä, Lorch, \& Kaakinen, 2002; Kaakinen \& Hyönä, 2008). In a related way, Radach, Huestegge, and Reilly (2008) argued that global factors (task demand and context) affect local eyemovement control by showing that words in a sentence presented in isolation are processed differently from words in the same sentence when it is preceded by an expository context. In their proposal and in further developments of this idea (Huestegge \& Bocianski, 2010), the role of global factors is confined to higher order contextual influences rather than visually driven oculomotor programs. Furthermore, the scanpath measure, defined as a time-locked spatial trajectory of saccadic eye-movements over text and proposed by von der Malsburg and Vasishth (2011) and von der Malsburg, Kliegl, and Vasishth (2015), demonstrates the merit of considering an entire line of text for establishing similarities and individual differences in linguistic processing.

While virtually unexplored in research on oculomotor control during reading (but see above), the notion of programs or schemas concurrently operating at different scales is central to the motor control theory. Schmidt's schema theory of motor control (Schmidt, 1975) divides motor programming of discrete actions into two components: the generalized motor program and the schema (Sherwood \& Lee, 2003). The generalized motor program (GMP) is responsible for invariant parameters of motor movements, such as sequence order and timing, i.e., GMP controls the general form of an action. The schema is the adjustment of an action to be performed within a specific environment (Schmidt, 2003) and is responsible for scaling of an action to match the environmental demands. The classic example 
of these two components of motor programing is when one writes her name in an 1-inch box and then again to fill a 3 -foot box. Although the absolute size is different, the relative movements of writing are the same resulting in signatures that look similar in shape and writing style, but differ in size. Therefore, in the aforementioned example, the relative temporal and kinetic parameters controlled by GMP are invariant (e.g., relative speed and spatial trajectories in handwriting), but the absolute parameters (e.g., movement amplitude, and overall speed) are scaled up to match the schema imposed by a larger sized box (Schmidt \& Lee, 2013).

While GMPs are considered to be stable and acquired via prior practice, schemas are labile and learned as needed. For example, in studies where subjects are asked to coordinate arm reaching movements under force fields that change the characteristics of the arm, the motor planning of reach movements are at first distorted (Lackner \& Dizio, 1994, 1998; Shadmehr \& Mussa-Ivaldi, 1994). After multiple trials, participants adapt to the new dimensions of the environment, and as a result, the number of distorted trajectories is reduced. This adaptation relies on the schema part of motor planning that occurs simultaneously with GMPs and adjusts the invariant components in hand trajectories for specific force fields (Kawato, 1999).

We propose that oculomotor control in reading involves a similar interaction between a relatively stable generalized motor program of word-to-word saccadic movements (labeled here a local level of planning), and the learned schema adjustable to the specific shape of the visual object to be processed or the task to be performed. Although different schemas are likely to exist for visually different objects (e.g., a sequence of a few words, a line of text, a passage, a page or a multi-page novel), this study investigates a schema specific to the processing of a sentence occupying one line on the screen (see below for our manipulation of tasks and the content of printed material).

Interestingly, the lengthening of saccades concomitant with progressive movement into the sentence finds a ready analogy in other motor sequences aimed at a rapid unidirectional motion through a spatial interval. For example, for a runner, optimal performance in a sprint race involves rapidly gaining speed through a series of initially short and increasingly lengthening steps (analogous to saccades) and then maintaining speed by making long equidistant steps (Hunter, Marshall, \& McNair, 2004; Kunz \& Kaufmann, 1981; Mann, Kotmel, Herman, Johnson, \& Schultz, 1984). Enomoto, Kadono, Suzuki, Chiba, and Koyama (2008) showed that medalist runners in the $10,000-\mathrm{m}$ race increased their step length by 5-7\% during the first $1200 \mathrm{~m}$ and effectively maintained this stride until the final stretch. Much like the program of pacing the steps when running is superimposed on the programs responsible for making each individual step, a global oculomotor program could be superimposed on other oculomotor programs that operate on a local word level.

Prior work investigating global components of oculomotor programs has focused on corpus data rather than experimental manipulations and on naturalistic reading materials in the form of sentences or paragraphs of text (cf. Kennedy \& Pynte, 2005 and references therein). This focus has left several questions unanswered. First, if a global line-level saccadic program is indeed in place, do readers implement it only when dealing with linguistic material (e.g., words in sentences) or any visual material (e.g., strings of symbols)? This question is important for establishing whether-in this particular phenomenon-the oculomotor system caters to specific linguistic goals or to more generic, non-semantic demands of processing letterlike information. There is some evidence that global influences on the oculomotor program are confined to linguistic materials, since Kuperman et al. (2010) did not find line-dependent modulations of fixation durations when readers were scanning meaningless $\mathrm{z}$-strings. However, it remains to be seen whether word position in line affects saccade amplitudes in meaningless reading (not reported in Kuperman et al., 2010) or in reading of other syntactically ill-formed stimuli (e.g., sequences of randomly shuffled words).

Second, does the oculomotor program primarily affect the "where" component of eye-movement planning and execution, or their "when" component? In other words, is the program's goal to optimize the uptake of information by regulating the distribution of saccades in space, or by regulating fixation durations, or both? Kuperman et al.'s data show evidence for the program in both the spatial and temporal domain. Yet, the two measures may be correlated since an increase of incoming saccade length usually entails longer subsequent fixation durations. Therefore, experimentally controlled stimulus material can shed further light on the relative contributions of the where and when components to a global oculomotor program.

\section{Present study}

To further examine the nature of global oculomotor control, the present study focuses on the start-up effect of word position in sentence on eye-movements. The central questions, therefore, are (1) whether the start-up effect is predicated on generic visual or specific linguistic characteristics and (2) whether the start-up effect is specific to spatial characteristics (saccade fixations) or temporal (durations of saccades) or both measures. We assessed eye movements while participants read stimuli in the following conditions. 
First, in a Nonequal length condition, we created three stimulus types comprising lines of character strings with variable length [see $(1 \mathrm{a}-\mathrm{c})$ below]: regular sentences (Sentence); strings of words that were shuffled and thus devoid of syntactic meanings (Shuffled); and z-strings, i.e., strings of identical symbols $z$ (Z-string). The number of characters (as well as capitalization and punctuation) in shuffled words and z-strings were matched one-to-one with the naturally varying word lengths in the regular sentences. In the shuffled condition (1b), the chosen words were novel but were matched in number of letters (word length) to words in the original sentence (1a): we did not reuse the original words to avoid repetitive exposure to the stimuli and to enable the word-by-word length matching.

(1a) The ducks swim in the pond near the bridge. (Sentence)

(1b) Bay chase trim to fly turn belt ear chills. (Shuffled)

(1c) Zzz zzzzz zzzz zz zzz zzzz zzzz zzz zzzzzz. (Zstring)

This three-level manipulation enables us to consider word position effects on fixation durations and saccade amplitudes (1a) in typical sentence reading, (1b) in a condition that shaves off the top-down (semantic and syntactic) support from the syntactic structure of a sentence while preserving the visual and lexical nature of its elements (Shuffled) and, (1c) in a condition that removes linguistic substance while preserving a typical sentence's visual layout (Z-string). Similar comparative manipulations were made in prior reading experiments that used z-strings (cf., Rayner \& Fischer, 1996; Rayner \& Raney, 1996; Vitu, O'Regan, Inhoff, \& Topolski, 1995) or Landolt circles (cf., Corbic, Glover, \& Radach, 2007; Williams \& Pollatsek, 2007) for meaningless visual inspection. Schad, Nuthmann and Engbert in 2010, also used sequences of shuffled words to remove semantic context while at the same time retaining lexical content.

Second, in an Equal length condition [see $(2 \mathrm{a}-\mathrm{c})$ for examples], we created the same three stimulus types (Sentence, Shuffled, Z-strings) with the restriction that all words (or strings) in a given sentence had the same length (4, 5,6 or 7 characters).

(2a) Most tiny dogs want love more than free food. (Sentence)

(2b) Frog fuzz goal hole jaws kiwi nuts plug fake. (Shuffled)

(2c) Zzzz zzzz zzzz zzzz zzzz zzzz zzzz zzzz zzzz. (Z-String)

A sentence composed of words of equal length reduces the expected variability in saccade amplitudes because of a uniform distance between the positions within words where most fixations land (the preferred viewing position), i.e., close to the word center (McConkie, Kerr, Reddix, \& Zola, 1988; Rayner, 1979; Vitu, O’Regan, \& Mittau, 1990). Therefore, this condition was intended to disentangle global oculomotor effects of word position on fixation durations from those on saccade amplitudes. That is, if the start-up effect is primarily driven by the "when" component of oculomotor control, equidistant word centers should yield a sentence-initial increase in fixation durations but not in saccade amplitudes. Alternatively, if the start-up effect is an inherent element of the "where"component, we may observe an increase of saccade amplitudes on sentence-initial words despite the equidistance of word centers.

In sum, two critical predictions for the start-up effect derive from observations of Pynte and Kennedy (2006) and Kuperman et al. (2010). The first prediction is that the amplitudes of the incoming saccades will undergo a steep increase in the beginning of a sentence with varying word lengths and then will increase at a much slower rate, if at all, for the remainder of the sentence. The second is that fixation durations will increase as the eyes move forward over the first few words of the sentence, then no such increase takes place in the middle region of the sentence, and another linguistically conditioned increase in fixation duration is observed in the sentence-final region. Our experiment investigates whether either of the hypothesized patterns will emerge if the inspected material lacks some or all linguistic content and if saccade amplitudes are restricted in range (Equal versus Nonequal word length).

\section{Methods \\ Participants}

Thirty-eight participants (31 females, 7 males) were recruited from the convenience pool of undergraduate students at McMaster University. Participants were compensated by course credit. The following were self-reported by all participants: each participant was a native speaker of English, had normal or corrected to normal vision and none had been diagnosed with a reading or learning disability.

\section{Apparatus}

Eye movements were recorded with an EyeLink1000 eyetracker, manufactured by SR Research Ltd. (Kanata, ON, Canada). The eyetracker is an infrared video-based tracking system combined with hyperacuity image processing. The eye movement camera and a conjoined 
infrared illuminator was mounted on a desktop beneath the stimulus display. The recording was monocular (right eye). The camera sampled pupil location and pupil size at a rate of $1000 \mathrm{~Hz}$. A chin support and forehead rest was used to stabilize participants' gross head movements. The stimuli were presented on a 19-in screen with a refresh rate of $60 \mathrm{~Hz}$ and a 1600-900 resolution. The average gaze position error of the EyeLink 1000 is $<0.05^{\circ}$, while its resolution is $0.01^{\circ}$ (root mean square error), with a microsaccade resolution of $0.01^{\circ}$.

\section{Stimuli}

We crossed two experimental manipulations to obtain a 3 (Sentence vs Shuffled vs Z-string) $\times 2$ (Nonequal vs Equal) factorial design. ${ }^{1}$ Each stimulus in the Equal condition consisted of words (and strings) of the same length, which varied from 4 to 8 characters. A small percentage of stimuli were allowed to deviate from the uniform length in the sentence by one character. Due to the difficulty of constructing equal-length sentences with 8-character words, only a few items were produced and did not yield stable results. In what follows, we only report sentences and strings with equal-length words that were between 4 and 7 characters, each represented by 10-12 stimuli. To reduce the duration of the experiment and participants' fatigue, we restricted the number of visually identical equal-length z-string stimuli to up to 6 for each word length. The Nonequal condition had no restriction on word length, which varied from 1 to 13 characters $(M=4.65$, $\mathrm{SD}=2.11)$. For compatibility between conditions, in our further analyses of the Nonequal condition, we only consider words between 4 and 7 characters, or $55 \%$ of all words.

In both Equal and Nonequal conditions, each sentence was matched word-by-word on word length (in characters), capitalization and punctuation with a shuffled sentence (consisting of existing words in a meaningless order) and with z-strings (consisting of word-like groups of $z$ symbols): for examples see $(1 \mathrm{a}-\mathrm{c})$ and $(2 \mathrm{a}-\mathrm{c})$ above. Due to a programming error, two sentences in the Nonequal condition did not have a matching $\mathrm{z}$-string in our stimulus list. We analyzed our data with and without removing the two sets of sentences and shuffled words that did not have a z-string match. Since the results demonstrated the same critical patterns, in what follows we report the full dataset. Table 1 summarizes the number of sentences and fixations in each condition. All sentences and sequences of randomly

\footnotetext{
1 A fourth gaze-contingent condition was implemented too, but failed to yield results due to a programming error in defining the gaze contingent trigger in the Experiment Builder software. The removal of this condition does not affect the other non-gaze contingent conditions and is therefore is not reported further.
}

shuffled words are available in the Online Supplementary materials.

All sentences (and their counterparts in other conditions) were limited to 90 characters in length and did not exceed one line on the computer screen. An average sentence in the Equal condition contained 7.2 words, and in the Nonequal condition 9.9 words. All sentences were displayed in size 18 Courier New font. One degree of the visual angle subtended approximately 3.3 characters. The order of sentences was randomized for each participant.

\section{Procedure}

Prior to presentation of the stimuli, the eye-tracker was calibrated using a series of 9 fixed targets distributed around the display, followed by a 9-point accuracy validation $\left(<0.05^{\circ}\right)$. Calibration was monitored throughout the experiment and was repeated after any breaks or whenever the experimenter judged necessary. Participants were instructed to "read all of the different types of strings as if [they] were reading a normal sentence". No comprehension questions were provided due to a small percentage of regular sentences among our stimuli. Participants were not informed that some sentences had equal-length words. As Table 2 below demonstrates, eye-movement patterns in regular sentences were compatible with ones reported for sentence reading for comprehension. Participants could take a break at any point during the experiment.

All participants were tested on the same set of stimuli. This within-subjects design offered additional control over individual differences in oculomotor, visual, cognitive and reading abilities and skills. Each trial began with a screen containing a fixation point positioned 26 pixels to the left of the first character in the sentence, which appeared when the experimenter pressed a button. Participants were limited to $10 \mathrm{~s}$ to complete the reading of the stimulus sentence. After they had read the sentence, participants looked at a gray, gaze contingent box $(100 \times 100$ pixels $)$ to initiate the next trial. The gaze contingent box removed the necessity to coordinate hand movements and eye-movements to move to the next trial. The duration of the eyetracking experiment did not exceed $60 \mathrm{~min}$.

\section{Data processing and analyses}

The unit of analysis was a word in a regular or shuffled sentence, or a sequence of z-symbols in a z-string. The original eye-movement record consisted of 48,382 data points. A removal of all skipped units reduced the dataset to 36,263 data points. Skipping rates are reported per condition in Table 2. Skipping rate of $29 \%$ in regular sentences with Nonequal word length is compatible with the skipping rate typically observed in skilled reading 
Table 1 Number of sentences and fixations (after trimming) per experimental condition

Table 2 Descriptive statistics per condition of skipping rate (in \%) for the entire dataset; regression rate (in \%) for all words that were fixated at least once; and incoming saccade amplitudes (in degrees) and single fixation durations (in $\mathrm{ms}$ ) for the subset of words fixated exactly once

\begin{tabular}{llllllcr}
\hline Condition & \multicolumn{2}{l}{$\mathrm{N}$ strings } & & & $\mathrm{N}$ fixations \\
\cline { 2 - 3 } & Sentence & Shuffled & Z-string & & Sentence & Shuffled & Z-string \\
\hline Equal: word length 4 & 10 & 10 & 5 & & 991 & 878 & 195 \\
Equal: word length 5 & 12 & 12 & 6 & & 1054 & 1117 & 236 \\
Equal: word length 6 & 10 & 10 & 5 & & 839 & 775 & 232 \\
Equal: word length 7 & 11 & 11 & 6 & & 848 & 793 & 211 \\
Nonequal & 50 & 50 & 49 & & 2460 & 2435 & 1547 \\
\hline
\end{tabular}

\begin{tabular}{|c|c|c|c|c|c|c|}
\hline \multirow[t]{2}{*}{ Condition } & \multirow[t]{2}{*}{ Skipping rate } & \multirow[t]{2}{*}{ Regression rate } & \multicolumn{2}{|c|}{ Saccade amplitude } & \multicolumn{2}{|c|}{ Single fixation duration } \\
\hline & & & Mean & SD & Mean & SD \\
\hline Nonequal: sentence & 29 & 7 & 2.50 & 0.85 & 227 & 89 \\
\hline Nonequal: shuffled & 22 & 11 & 2.22 & 0.79 & 281 & 126 \\
\hline Nonequal: Z-string & 43 & 6 & 2.60 & 1.10 & 273 & 139 \\
\hline Equal: sentence & 13 & 9 & 2.44 & 0.79 & 247 & 99 \\
\hline Equal: shuffled & 9 & 10 & 2.33 & 0.75 & 298 & 122 \\
\hline Equal: Z-string & 35 & 5 & 2.80 & 1.16 & 275 & 140 \\
\hline
\end{tabular}

(Rayner, 1998). Skipping rate in sentences with equallength words was noticeably lower $(13 \%)$. We explain this discrepancy by a much lower number of often skipped function words in these sentences, as compared to their Nonequal length counterparts.

We further removed units in which the duration of the first fixation was shorter than $50 \mathrm{~ms}$ or longer than $1000 \mathrm{~ms}$. Regression rates per condition are reported in Table 2. Observed rates were not drastically different from the $10-15 \%$ regression rate reported as typical for proficient readers (Rayner, 1998). We also removed units that had a regressive incoming saccade (leaving 31,872 data points) and restricted the incoming saccade amplitude to the range of $1^{\circ}-6^{\circ}$ of visual angle (leaving 29,790 data points). Since saccade planning and execution is at the core of our investigation, we further opted for only considering units that elicited a single fixation. Multiple fixations on a unit are often a result of a mislocated initial saccade to the word and a concomitant spatial correction (cf., McConkie et al., 1988; Rayner, 1998). The resulting dataset consisted of 24,248 data points, with 10,454 data points in the Equal condition and 13,794 in the Nonequal condition. For comparability with Kuperman et al. (2010), we removed fixations and saccades of the initial and final word in each sentence or string; and we only considered words in the length range from 4 to 7 characters in the Nonequal condition to match the corresponding word lengths in the equal one. This left us with 8169 and 6442 data points in the equal and Nonequal conditions, respectively. This data pool contained single fixations that were made during any reading pass. Analyses were then conducted on a data pool that was further constrained to only first-pass single fixations. Those analyses replicate all the critical patterns observed in reading and are not reported here. Table 2 reports the breakdown of observations per each cell in our design. Both single fixation durations and saccade amplitudes in regular sentences were comparable to parameters typically observed in reading for comprehension.

In our analyses, we focused on the effect that word (or string) position in the line has on eye-movements to that word. For comparability between sentences with different numbers of words, we calculated the relative word position as a ratio of the ordinal word position in the line to the total number of words in that line. Following our hypotheses and the analyses in Kuperman et al. (2010), we examined relative word position effects on incoming saccade amplitudes (in degrees of visual angle) and on single fixation durations (i.e., duration of the one and only fixation to the word, in ms). Table 2 presents descriptive statistics for the dependent variables.

The functional relationship between word position and the dependent variable was expected to be nonlinear in nature and to come with radically different slopes for different subranges of the independent variable (e.g., a steep increase of saccade amplitudes in the sentence-initial region and little to no increase in the remainder of the sentence). This requires a separate estimation of statistical significance for different segments of the functional curve and can be achieved via the analytical technique of segmented regression, which we adopt here. This technique takes as its input the starting values for one or more expected breakpoints. It makes inferential estimates of the values of the independent variable that serve as optimal breakpoints for the functional curve, as well as the slopes 
Fig. 1 Segmented regression lines (dashed) fitted to incoming saccade amplitudes for sentences (left), shuffled sentences (middle) and z-strings (right) with words of Non-equal length. Dotted lines represent $95 \%$ confidence intervals. Gray dots show mean values for deciles of relative word positions: error bars represent 1 standard error. Gray lines show partial effects of word position as estimated by the linear mixed-effects models

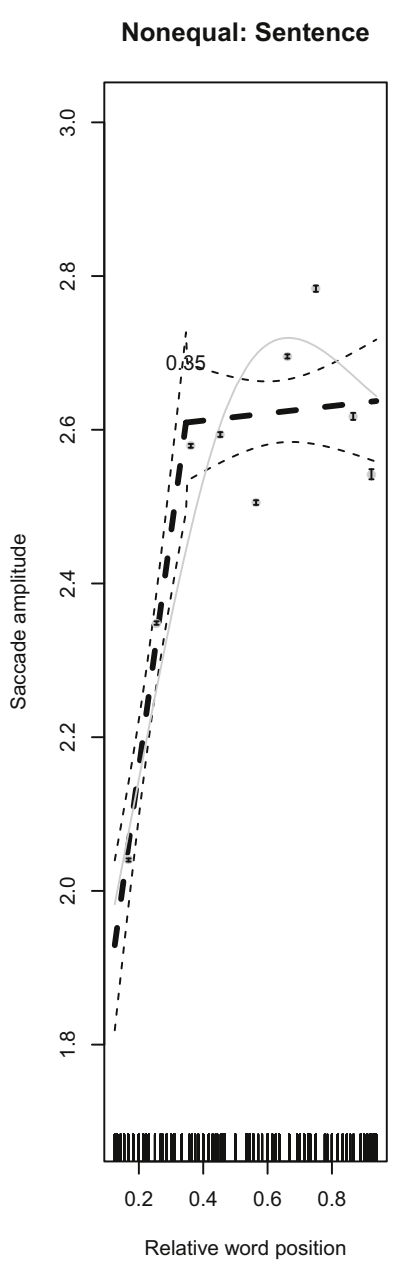

of each resulting segment. ${ }^{2}$ For instance, the output of a segmented regression model with one breakpoint has two segments and reports an intercept $\left(b_{0}\right)$, a slope for the first segment $\left(b_{1}\right)$ as well as an adjustment $\left(b_{2}\right)$ to the first segment's slope that characterizes the slope of the second segment. The absolute slope of the second segment is, therefore, estimated as $b_{1}+b_{2}$. Due to the difficulty in estimating the degrees of freedom for segments beyond the first one, only $t$ values and no $p$ values are provided for those segments with the model output. We considered slopes with the $|t|$ value $>2$ to be statistically reliable at the 0.05 threshold. Segmented regression enables us to evaluate whether the start-up region in the sentence does indeed show a statistically reliable increase in saccade amplitudes

\footnotetext{
${ }^{2}$ While segmented regressions estimate breakpoints as discrete parameters by definition, we make no inferences about whether respective transitions between regression slopes are discrete or gradual in nature (for a gradual modeling of same patterns, see mixed effects models with cubic splines in the Supplementary Materials and Fig. 1). Accordingly, discrete breakpoints in our analyses do not necessarily imply qualitative differences between processes in different segments.
}
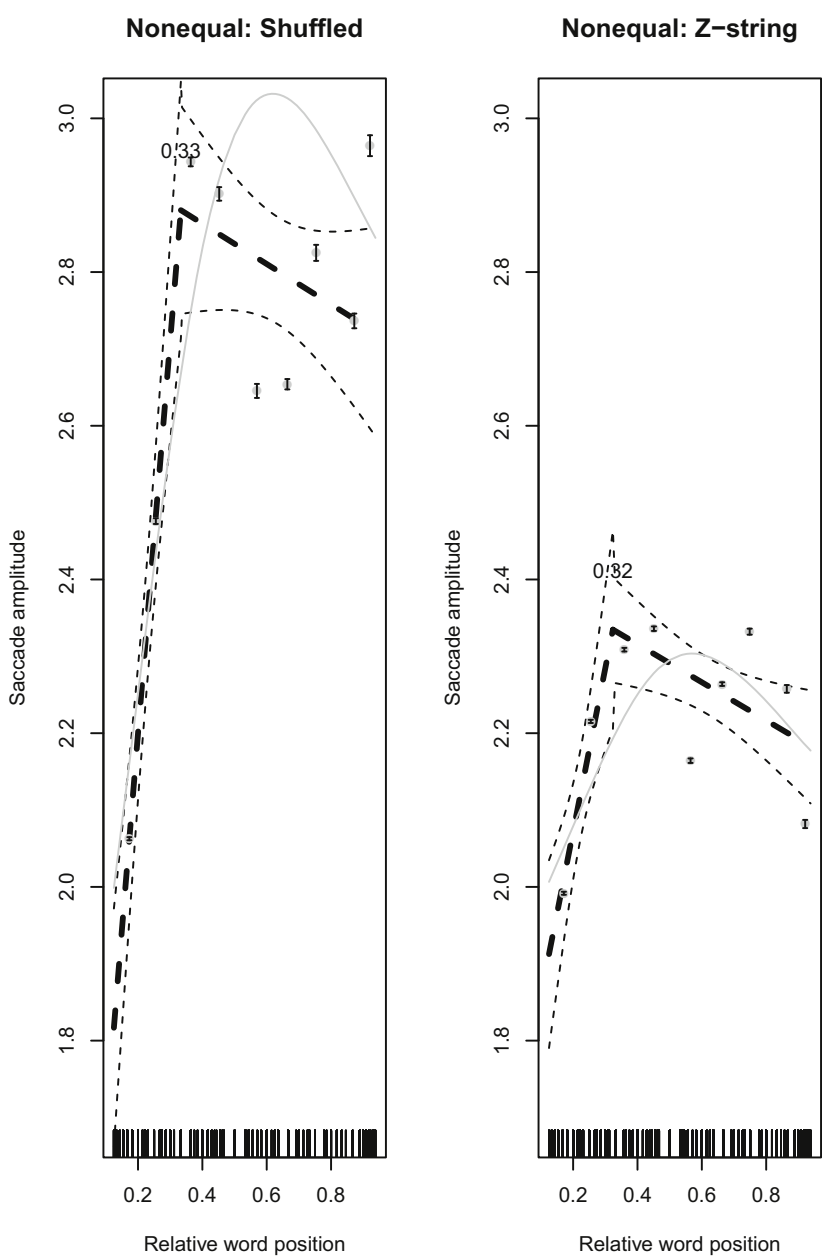

or fixation durations and also indicates the breakpoint where a predicted plateau is reached.

Our primary interest was in the shape of the functional relationship that word position in line had with the dependent variable in each condition, rather than how those conditions differed from one another in the intercept or slopes of their respective regression lines. For this reason, we fitted separate sets of statistical models to each dependent variable (saccade amplitude or single fixation duration) and every condition with relative word position as predictor. Specifically, each set of models included a multiple linear regression, a segmented regression model with one breakpoint (i.e., two segments) and a segmented regression model with two breakpoints (i.e., three segments). The within-participant nature of the experiment provided a natural control for individual variability in perceptual, oculomotor or linguistic ability across conditions. Version 3.2.2 of the $\mathrm{R}$ programming language was used for all analyses (R Core Team, 2015). The models' goodness-of-fit indices were compared using the modelcomparison function anova() implemented in R. Only the best-fitting models are reported below for each dependent variable. 
Table 3 Segmented regression models for sentences, shuffled sentences and z-strings in the Nonequal condition, with saccade amplitudes as a function of word position

\begin{tabular}{lrlrl}
\hline & Estimate & Std. error & $t$ value & $\operatorname{Pr}(>|t|)$ \\
\hline Sentence & & & & \\
Intercept & 1.545 & 0.109 & 14.149 & $<0.001$ \\
WordPos.Segment 1 & 3.075 & 0.455 & 6.758 & $<0.001$ \\
WordPos.Segment 2 & -3.028 & 0.47 & -6.448 & \\
Shuffled & & & & \\
Intercept & 1.178 & 0.158 & 7.435 & $<0.001$ \\
WordPos.Segment 1 & 5.112 & 0.683 & 7.484 & $<0.001$ \\
WordPos.Segment 2 & -5.374 & 0.711 & -7.562 & \\
Z-string & & & & \\
Intercept & 1.647 & 0.13 & 12.686 & $<0.001$ \\
WordPos.Segment 1 & 2.125 & 0.573 & 3.711 & $<0.001$ \\
WordPos.Segment 2 & -2.373 & 0.581 & -4.081 & \\
\hline
\end{tabular}

Breakpoints for sentence, shuffled, and z-strings conditions are 0.35 , 0.33 and 0.32 . Respective $R^{2}$ are $0.06,0.08$ and 0.02

A current implementation of the package segmented (Muggeo, 2003, 2008) does not allow for including random effects. To make sure we account for the within-participant repeated-measures nature of our data, we further supplemented sets of regression models outlined above by fitting linear mixed-effects multiple regression models to the same set of dependent variables, while using both the maximal random-effects structure and word length as an additional covariate. As discussed below, these models replicated the qualitative nature of the functional curves that we obtained with the segmented regression solutions. In the "Supplementary Materials", we report these models with $p$ values obtained using the Satterthwaite's approximation for degrees of freedom as implemented in lmerTest package v. 2.0 (Kuznetsova, Brockhoff, \& Christensen, 2015).

\section{Results}

\section{Incoming saccade amplitude: Nonequal condition}

For stimuli with words or strings of Nonequal lengths, the relationship between incoming saccade amplitudes and relative word position showed consistency across all conditions. In sentences, in shuffled words as well as in z-strings, the function is best described as a steep initial increase in saccade amplitudes, followed by a weaker increase (Sentences) or no increase (Shuffled and Z-strings). For illustrative purposes, Fig. 1 shows average saccade amplitudes estimated for deciles of relative word position, but note that the models were fitted to non-aggregated data.

The statistical analyses confirmed the non-linear trend of saccade amplitudes. Segmented regression models with one breakpoint performed reliably better than ordinary regression models or segmented regressions with two breakpoints (see Table 3 for model specifications). Consistent across all reading conditions, the best fitting models identified a breakpoint at around one-third of the overall sentence length, with the slope in the second segment being significantly flatter than the slope in the sentence-initial segment. Table 3 further indicates similarities in the absolute intercepts and slopes for the first segment across text types. Overall, these results suggest that the characteristic pattern of the saccade amplitude distribution over a line is not specific to syntactically well-formed sentences, nor to asyntactic lexical material (implemented in the Shuffled condition), but generalizes over any reading material that has word-like visual features (Z-strings).

We further confirmed the characteristic patterns by fitting linear mixed effects multiple regression models to incoming saccade amplitudes, with word position as a nonlinear predictor (modeled using restricted cubic splines with 3 knots), word length as a linear predictor, as well as random intercepts for participants and items and by-participant random slopes for word position (see Supplementary Materials, Tab. S1, S2 and S3 for full model specifications and results). Partial effects of word position (shown as solid gray lines in Fig. 1) were qualitatively similar to the fits provided by segmented regression models.

\section{Incoming saccade amplitude: equal condition}

Stimuli with words or strings of equal length posit an obvious restriction to the variability of saccade amplitudes. If readers are perfectly attuned to word lengths and select the preferred viewing position as their saccadic target, one would expect these stimuli to elicit saccades of equal amplitudes across the entire line. One can also expect overall longer saccade amplitudes for stimuli with long as compared to short words.

Figure 2 confirms the latter prediction. Sentences, shuffled words and z-strings containing longer words came with longer saccades. Critically, however, the figure also revealed an increase in saccade amplitudes, rather than a flat line for all types of stimuli and for most word lengths. The increase was observed either in the beginning of a line (sentences with 7-character words) or across the entire line. Statistical significance of the effects reported by regression models varied across types of sentences, but the effect of word position on incoming saccade amplitude was 
Fig. 2 Segmented or linear regression best-fit lines fitted to incoming saccade amplitudes for sentences (left), shuffled sentences (middle) and z-strings (right) with words of equal length

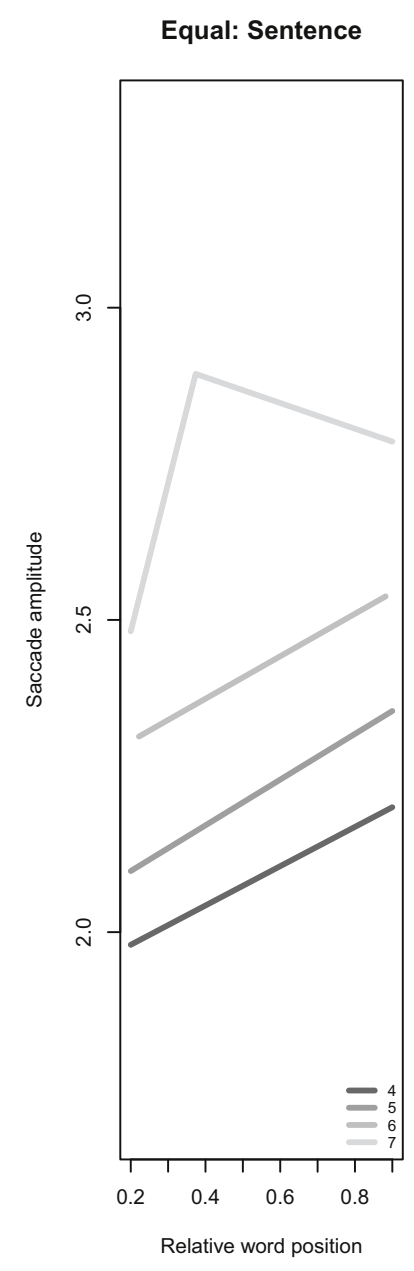

Equal: Z-string

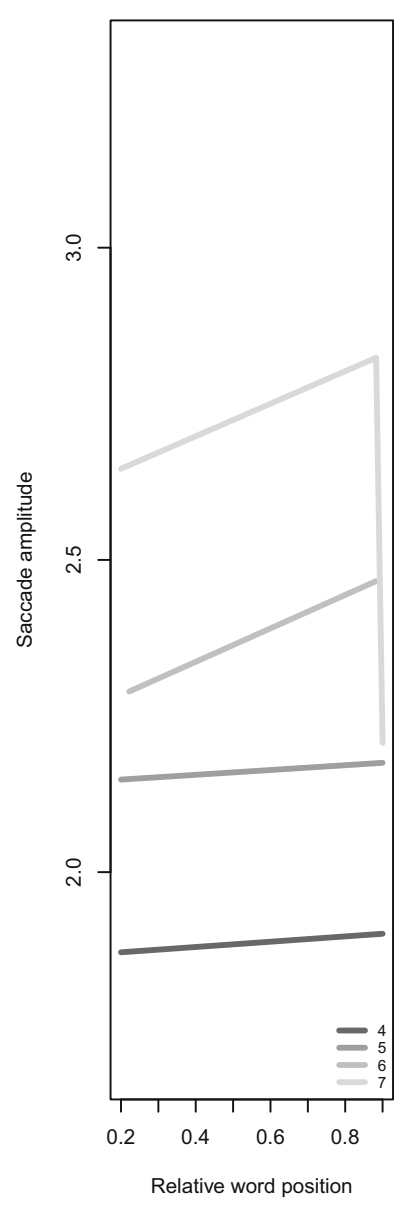

Table 4 Segmented and linear regression models for sentences with equal-length words (condition Equal sentence), with saccade amplitudes as a function of word position

\begin{tabular}{llclrr}
\hline Word length & Predictor & Estimate & Std. error & $t$ value & $\operatorname{Pr}(>|t|)$ \\
\hline 4 & Intercept & 1.917 & 0.051 & 37.576 & $<0.001$ \\
& WordPos & 0.315 & 0.085 & 3.702 & $<0.001$ \\
5 & Intercept & 2.025 & 0.051 & 39.410 & $<0.001$ \\
& WordPos & 0.366 & 0.087 & 4.205 & $<0.001$ \\
6 & Intercept & 2.238 & 0.060 & 37.262 & $<0.001$ \\
& WordPos & 0.340 & 0.101 & 3.373 & 0.001 \\
7 & Intercept & 1.969 & 0.407 & 4.833 & $<0.001$ \\
& WordPos & 2.530 & 1.557 & 1.625 & 0.105 \\
& WordPos.Segment 2 & -2.729 & 1.564 & -1.745 & \\
\hline
\end{tabular}

estimated as significant (at the 0.05 threshold) for sentences of all lengths except seven-character words, which showed a trend $(p=0.105)$. Table 4 reports segmented regression model specifications for equal word-length sentences, while model outputs for shuffled words and z-strings are reported in Supplementary Materials (Tab. S4).

In sum, the start-up effect in saccade amplitudes in the Equal condition is less expressed than in the Nonequal condition. As outlined above, this reduction of the effect is expected due to the equidistant word centers, i.e., saccade targets. Yet, it is noteworthy that we observed increasing saccade amplitudes over the line of a text at all. This finding indicates that the start-up effect is a dominant element in the 'where' component of eye movements as it supports the notion of an oculomotor program that aims at guiding the eyes in shorter steps at the beginning than in the middle or the end of a line. In our data, most sentences, shuffled strings and z-strings with words of equal lengths 
Fig. 3 Mean single fixation durations estimated for deciles of relative word position, broken down by Nonequal (left) and Equal (right) conditions, and sentences (solid line), shuffled strings (dashed) and z-strings (dotted). Error bars represent 1 standard error
Nonequal

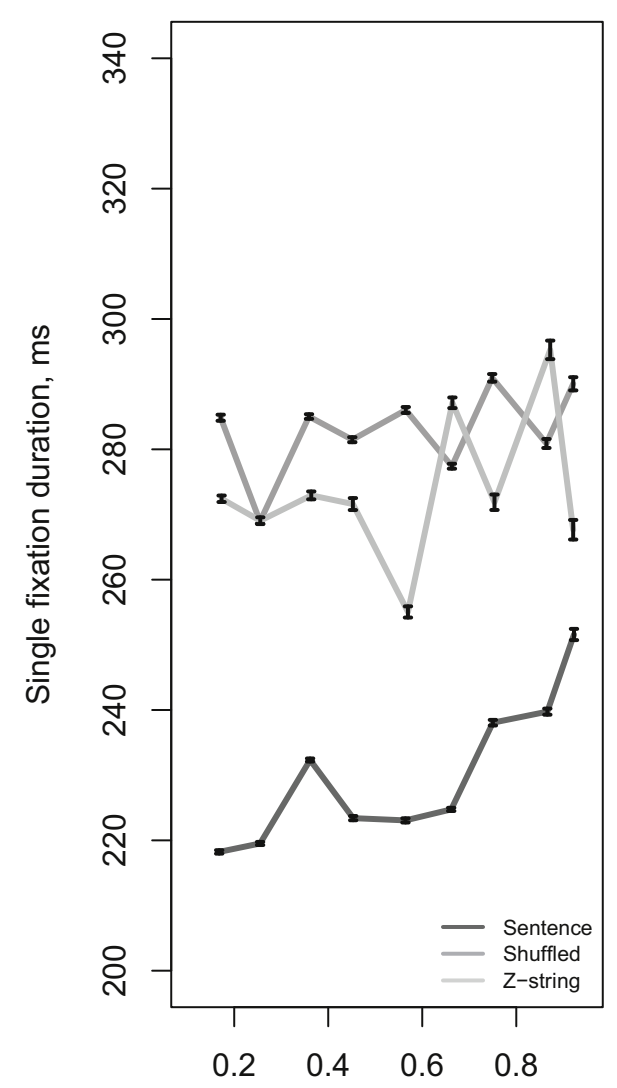

Relative word position
Equal

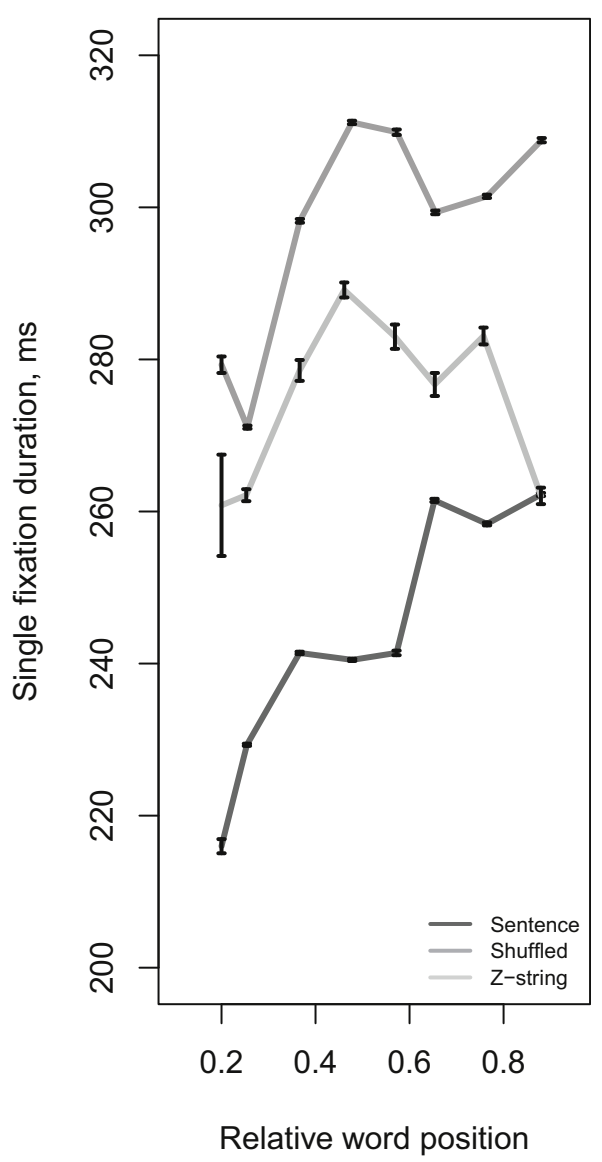

elicited a constant sentence-long increase in saccade amplitudes. An exception are sentences with long words of seven characters, which have a breakpoint and a change in the effect size after the initial increase (Fig. 2, left panel).

\section{Single fixation durations}

Figure 3 summarizes mean single fixation durations over deciles of relative word position for the Nonequal and Equal conditions. A reliable increase in fixation durations was observed in sentences with words of variable and fixed lengths (Nonequal sentence and Equal sentence), as indicated by linear mixed-effects regression models with word position as a critical linear predictor (see Supplementary Materials, Tab. S5-S10) or a non-linear predictor (modeled using restricted cubic splines with 3 knots, not shown). A reliable increase in fixation durations was also observed in sequences of shuffled equal-length words (Equal shuffled). While the magnitude of these effects varied across conditions, in all cases, an increase in fixation durations was observed over initial words of the sentence (start-up), followed by a plateau and an additional increase over final sentence words (wrap-up).

Conversely, shuffled and Z-strings in the Nonequal condition and Z-strings in the Equal condition did not show a consistent relationship with word position in sentence. This discrepancy in durational patterns supports the findings of Kuperman et al. (2010) who observed a sentence-long increase in fixation durations in regular sentences but not in Z-strings. Importantly, the discrepancy between conditions runs counter to the uniformity of the effect-and especially its start-up component - that we found in saccade amplitudes in all types of stimuli (cf., Fig. 1). We conclude that a start-up or a wrap-up inflation in fixation durations is effectively confined to linguistically meaningful stimuli (i.e., the ones which present lexical material, with or without a wellformed syntactic structure) and is not solely contingent on visual characteristics of the input. Taken together, these results suggest that durational measures do not mirror saccadic patterns (as observed in Kuperman et al., 2010) and support the conclusion that the start-up effect driven by the visual system is inherent to the 'where' rather than the 'when' component of oculomotor control. 


\section{Discussion}

Much research on eye-movement control during reading has focused on the nature and degree of the mutual constraint that the visual, oculomotor and linguistic systems impose on one another. Most work in the field so far investigated an oculomotor program that operates within a reader's visual span. Such a program is arguably responsible for the recognition of a fixated word as well as for the processing and the eyes' progression through words in the parafovea. Following up on prior work by Pynte and Kennedy (2006) and Kuperman et al. (2010), though, this paper argues that reading engages several oculomotor programs that operate simultaneously at different spatial scales. Specifically, we examined the claim that the ballistic movements of the eye follow a regular pattern when moving along a line of text, optimized such that the average speed of processing is neither too low (leading to inefficiency) nor too high (leading to a costly re-inspection of the text). As we discuss in the "Introduction", reaching an optimal speed in motor tasks that involve propelling of a body through space in a series of stops (fixations) and gos (saccades) is achieved through a sequence of initially short and gradually increasing steps, followed by a relatively stable step length (Enomoto et al., 2008; Hunter et al., 2004; Kunz \& Kaufmann, 1981; Mann et al., 1984). Corpus studies of eye-movements in reading revealed exactly this "start-up" effect as function of word position in a line, i.e., a marked increase in saccade amplitudes (Kuperman et al., 2010; Pynte \& Kennedy, 2006) and fixation durations (Kuperman et al., 2010) over the initial few words of the sentence. The present paper advances the study of the proposed line-wide oculomotor program by interrogating its nature and magnitude using stringent experimental control and by eliminating layers of information, from higher order linguistic cues (context, syntax and semantics) to lower level visual cues (uniform word length).

This study addresses two main questions. First, is the start-up effect truly oculomotor in character and thus existent during scanning of any visual layout of groups of symbols, or is it a phenomenon that is restricted to the processing of linguistic information? We addressed this question by presenting strings that were matched on all visual characteristics, but varied from syntactically and lexically well-formed sentences to sequences of existing words shuffled in a meaningless order to sequences of meaningless $\mathrm{z}$-strings. Thus, the critical indication of whether the start-up effect is purely oculomotor is in whether it changes qualitatively as a function of the incremental removal of the top-down contextual support in the Shuffled condition and of all linguistic content in the Z-string condition.
Second, we were interested in whether the start-up effect is primarily driven by the "where" or the "when" component of eye-movement planning and execution, or whether it is equally present in both. Our prior research (Kuperman et al., 2010) showed a gradual increase in both saccade amplitudes and fixation durations over the sentence beginning, and so the two behavioral outcomes were not teased apart. In the present study, we reduced the expected variability in saccade amplitudes by creating sets of sentences (and sentence-like strings) that contained words of equal length. Given the evidence for some independence of the 'where' and 'when' component in oculomotor control (Balota et al., 1985; Balota \& Rayner, 1983; Ehrlich \& Rayner, 1981; Hirotani et al., 2006; McConkie et al., 1988; Morris, Rayner, \& Pollatsek, 1990; Morrison, 1984; Rayner, Binder, Ashby, \& Pollatsek, 2001; Rayner, Chace, Slattery, \& Ashby, 2006; Underwood, Clews, \& Everatt, 1990), equal word lengths constrained the ground for a start-up effect in saccade amplitudes, but maintained it for fixation durations. However, we also reasoned that the start-up effect in saccade amplitudes might prevail even against equidistant saccade targets if it is inherently driven by the 'where' component.

The observed data patterns shed light on both questions. We found a consistent start-up effect in saccade amplitudes in sentences that naturally varied in length, as well as in associated shuffled words and z-strings (Fig. 1). A steep increase in saccade amplitudes over the first third of a sentence was followed either by a flat line or a less steep increase. That the effect persists across all types of written material provides clear evidence that the nature of the effect is purely visual, and not linguistically constrained.

Our manipulation of uniformity in word length artificially restricted the saccade range, because it kept constant the distance between preferred viewing positions across the entire sentence. To continue with the runner's metaphor, this manipulation is equivalent to placing marks at equal intervals along the entire race track and suggesting that, at each step, the runner's feet land on the mark. As expected, the start-up effect in saccade amplitudes was strongly attenuated in equal-length stimuli (Fig. 2). Nevertheless, the overall trend suggests that readers still lengthen their saccades, and sometimes reliably so, even though preferred landing positions were equidistant. In sum, we conclude that the oculomotor program operating at the line scale is revealed in the sentence-initial increase in saccade amplitudes, is contingent on the visual layout of a string rather than its linguistic content and is observed even in conditions where such increase is artificially suppressed.

Another finding was that the start-up effect on fixation durations, as well as the wrap-up effect, are specific to processing of linguistic information and are not found in 
visually similar but meaningless z-strings: this held true for both Equal- and Nonequal length stimuli and was consistent with observations of Kuperman et al. (2010). This suggests that the oculomotor program that operates over an entire line and is consistent across types of stimuli and length constraints is primarily geared towards the "where" component of reading and the visual properties of the printed texts. We also speculate that the "when" component, gauged via fixation durations, is primarily enmeshed with the word-to-word processing effort and is more indicative of the oculomotor program that operates on a smaller scale, i.e., within one's visual span.

The degree to which different oculomotor programs are dissociable on the level of saccades and fixation durations remains subject to future research. One promising approach to further delineate effects particularly at the global level are reading strategies, which have been shown to induce global changes in eye movement patterns. For instance, reading times are substantially slowed down when participants expect difficult compared to easy comprehension questions, or when they are proofreading a text compared to normal reading (Wotschack \& Kliegl, 2013; Wotschack, 2009). Such modulations of task demands at the level of readers rather than at the level of text materials (as in the present study) may well be suited to reveal further constraints of oculomotor programs.

Our hypothesis of a superposition of motor programs operating on both the local and global levels aligns well with the influential Schmidt's (1975) schema theory of motor control. Under this theory, the saccadic motor action that subserves local transitions from one word to another relies on largely invariant spatiotemporal and kinetic parameters which are characteristic of generalized motor programs (GMP). However, the motor output is further adjusted to the demands of a specific task, via a schema acquired through motor learning. In the present study, we propose that these global-level, learnable adjustments in saccade amplitudes and timing emerge when words are presented as part of a larger visual object (a line of text). We argue then that the patterns in saccade amplitudes that we observe as a function of word position are a reflection of this motor schema. We also speculate that Radach et al., (2008) finding of a processing difference between words in a sentence read in an isolation vs. words in the same sentence read as part of a passage is at least partly due to the adjustments caused by different oculomotor schemas, which are associated with the visual inspection of one-line sentences vs. text passages rather than the pure top-down influence of linguistic context. Similarly, a difference in schemas may contribute to the influence of task demands on eye-movement patterns (Kaakinen \& Hyönä, 2010; Wotschack \& Kliegl, 2013; Wotschack, 2009)
Our findings are also relevant in the realm of existing models and theories of reading, and their present accounts for a variety of eye movement patterns within the local visual span (Engbert \& Kliegl, 2001; Just \& Carpenter, 1980; Morrison, 1984; Reichle, Pollatsek, Fisher, \& Rayner, 1998). So far, eye guidance on a global level of sentences or paragraphs plays a subordinate role. Particularly our results regarding saccade amplitudes show that the consideration of such a global program is needed for a comprehensive account of oculomotor control in reading. Interestingly, current models of oculomotor control already implement components that might be able to capture global eye movement patterns, but have not yet been rigorously tested in this domain.

The serial E-Z Reader model, for instance, assumes that words are locally attended one at a time in a strictly serial fashion and that saccades towards the center of the next word are triggered by the progress of the current lexical process (Reichle et al., 1998; Reichle, Pollatsek, \& Rayner, 2006). Yet, a global pre-attentive stage following each saccade takes up information from the entire visual field and provides information such as the shape of a sentence or word boundaries. Global text properties and their spatial relation to the current eye position are, therefore, available at each fixation and may inform a global program that acts on top of local processes. In addition, E-Z Reader was recently augmented by a post-lexical integration stage that accounts for lexically and syntactically driven sentence wrap-up effects in fixation durations and regressions (Reichle et al., 2009). Our findings show that supplementing a model with saccade planning parameters driven by visual properties on sentence, line- or text-levels may be beneficial.

A global program is also plausible in the parallel framework of SWIFT, a model proposing that words within the perceptual span are processed in parallel while a random timer triggers saccades partly independently from lexical processing (Engbert et al., 2005). SWIFT implements parallel lexical processing as a dynamic field, in which activation of words first builds up based on visual, contextual and lexical cues and then decreases due to lexical completion. Words thereby compete as saccade targets according to the relative height of their momentary activation. The parallel-dynamic nature of SWIFT would account for the start-up effect in saccades if systematic changes of the activation field across the course of a sentence features nearer saccade targets at sentence initial than at middle or final positions (e.g., through an increase of context information or more pre-processing on later words). Further, a recent SWIFT implementation of a dynamic perceptual span proposed that lexical processing of a fixated word slows down in the presence of residual activation (i.e., incomplete lexical completion) on words left of the fixation (Schad \& Engbert, 2012). Since the 
probability of such residual activation increases with word position, fixation durations may increase over the course of a sentence.

It should be noted that these tentative proposals remain to be examined as a source of global eye movement patterns in models of oculomotor control. In fact, such tests provide a novel ground for model comparisons, which usually ignore criteria on a sentence-level. Finally, we believe that a model that simultaneously accounts for eye movements on both a local and a global level would substantially contribute to the understanding of the coordination between oculomotor control and word recognition.

In summary, we provide new evidence that eye-movement control in reading is performed at several scales. We further show that the global line-wide program that aims at quick and efficient progression of the eyes through a line of text is robust, relies on visual rather than linguistic information from the input and primarily regulates the spatial characteristics of motor planning. Furthermore, it appears to operate independently of the better studied local eyemovement control on the word level. We, therefore, conclude that the measurable behavioral indices of eyemovements in reading represent a superimposition of oculomotor control on several operational levels.

\section{Compliance with ethical standards}

Funding This work was supported by the funding from Natural Sciences and Engineering Research Council or Canada (NSERC) Discovery Grant 402395-2012, the Early Research Award from the Ontario Ministry of Research and Innovation, the National Institutes of Health NIH R01 HD 073288 (PI Julie A. Van Dyke), and the Canada Research Chair (Tier 2) award to Victor Kuperman. This work was also completed by Noor Al-Zanoon as an undergraduate thesis in the Cognitive Science of Language program at McMaster University (Hamilton, Ontario, Canada).

Conflict of interest All authors declare that there is no conflict of interest.

Ethics All procedures performed in studies involving human participants were in accordance with the ethical standards of the institutional and/or national research committee (McMaster Research Ethics Board, protocol 2011 165).

Informed consent Informed consent was obtained from all individual participants included in the study.

\section{References}

Balogh, J., Zurif, E., Prather, P., Swinney, D., \& Finkel, L. (1998). Gap-filling and end-of-sentence effects in real-time language processing: implications for modeling sentence comprehension in aphasia. Brain and Language, 182(61), 169-182.

Balota, D. A., Pollatsek, A., \& Rayner, K. (1985). The interaction of contextual constraints and parafoveal visual information in reading. Cognitive Psychology, 17(3), 364-390.
Balota, D. A., \& Rayner, K. (1983). Parafoveal visual information and semantic contextual constraints. Journal of Experimental Psychology: Human Perception and Performance, 9(5), 726-738. doi:10.1037/0096-1523.9.5.726.

Corbic, D., Glover, L., \& Radach, R. (2007). The Landoldt-C string scanning task as a proxy for visuomotor processing in reading: A pilot study. In Poster session presented at the 14th European Conference on Eye Movements.

Ehrlich, S. F., \& Rayner, K. (1981). Contextual effects on word perception and eye movements during reading. Journal of Verbal Learning and Verbal Behavior, 20(6), 641-655. doi:10.1016/ S0022-5371(81)90220-6.

Engbert, R., \& Kliegl, R. (2001). Mathematical models of eye movements in reading: A possible role for autonomous saccades. Biological Cybernetics, 85(2), 77-87. doi:10.1007/PL00008001.

Engbert, R., Nuthmann, A., Richter, E. M., \& Kliegl, R. (2005). SWIFT: A dynamical model of saccade generation during reading. Psychological Review, 112(4), 777-813. doi:10.1037/ 0033-295X.112.4.777.

Enomoto, Y., Kadono, H., Suzuki, Y., Chiba, T., \& Koyama, K. (2008). Biomechanical analysis of the medalists in the 10,000 metres at the 2007 World Championships in Athletics. New Studies in Athletics, 3, 61-66.

Hill, R. L., \& Murray, W. S. (2000). Commas and spaces: effects of punctuation on eye movements and sentence parsing. In A. Kennedy, R. Radach, D. Heller, \& J. Pynte (Eds.), Reading as a Perceptual Process (pp. 565-589). Amsterdam, Netherlands: Elsevier. doi:10.1016/B978-008043642-5/50027-9.

Hirotani, M., Frazier, L., \& Rayner, K. (2006). Punctuation and intonation effects on clause and sentence wrap-up: Evidence from eye movements. Journal of Memory and Language, 54, 425-443. doi:10.1016/j.jml.2005.12.001.

Huestegge, L., \& Bocianski, D. (2010). Effects of syntactic context on eye movements during reading. Advances in Cognitive Psychology, 6, 79-87.

Hunter, J. P., Marshall, R. N., \& McNair, P. J. (2004). Interaction of step length and step rate during sprint running. Medicine and Science in Sports and Exercise, 36(2), 261-271. doi:10.1249/01. MSS.0000113664.15777.53.

Hyönä, J., Lorch, R. F., \& Kaakinen, J. K. (2002). Individual differences in reading to summarize expository text: Evidence from eye fixation patterns. Journal of Educational Psychology, 94(1), 44-55. doi:10.1037/0022-0663.94.1.44.

Just, M. A., \& Carpenter, P. A. (1980). A theory of reading: From eye fixations to comprehension. Psychological Review, 87(4), 329-354.

Kaakinen, J. K., \& Hyönä, J. (2008). Perspective-driven text comprehension. Applied Cognitive Psychology, 22(3), 319-334. doi:10.1002/acp.1412.

Kaakinen, J. K., \& Hyönä, J. (2010). Task effects on eye movements during reading. Journal of Experimental Psychology. Learning, Memory, and Cognition, 36(6), 1561.

Kawato, M. (1999). Internal models for motor control and trajectory planning. Current Opinion in Neurobiology, 9(6), 718-727. doi:10.1016/S0959-4388(99)00028-8.

Kennedy, A., \& Pynte, J. (2005). Parafoveal-on-foveal effects in normal reading. Vision Research, 45(2), 153-168. doi:10.1016/j. visres.2004.07.037.

Kunz, H., \& Kaufmann, D. A. (1981). Biomechanical analysis of sprinting: decathletes versus champions. British Journal of Sports Medicine, 15(3), 177-181. doi:10.1136/bjsm.15.3.177.

Kuperman, V., Dambacher, M., Nuthmann, A., \& Kliegl, R. (2010). The effect of word position on eye-movements in sentence and paragraph reading. Quarterly Journal of Experimental Psychology, 63(9), 1838-1857. doi:10.1080/ 17470211003602412. 
Kuznetsova, A., Brockhoff, P. B., \& Christensen, R. H. B. (2015). lmerTest: Tests in Linear Mixed Effects Models. Retrieved from http://cran.r-project.org/package=lmerTest.

Lackner, J. R., \& Dizio, P. (1994). Rapid adaptation to Coriolis force perturbations of arm trajectory. Journal of Neurophysiology, 72(1), 299-313.

Lackner, J. R., \& Dizio, P. (1998). Gravitoinertial Force Background Level Affects Adaptation to Coriolis Force Perturbations of Reaching Movements. Journal of Neurophysiology, 80, 546-553.

Liversedge, S. P., Drieghe, D., Li, X., Yan, G., Bai, X., \& Hyönä, J. (2016). Universality in eye movements and reading: a trilingual investigation. Cognition, 147(3), 1-20. doi:10.1016/j.cognition. 2015.10.013.

Mann, R., Kotmel, J., Herman, J., Johnson, B., \& Schultz, C. (1984). Kinematic trends in elite sprinters. Proceedings of the International Symposium of Biomechanics in Sports (pp. 17-33). Del Mar, California: Academic Publishers.

McConkie, G. W., Kerr, P. W., Reddix, M. D., \& Zola, D. (1988). Eye movement control during reading: I. The location of initial eye fixations on words. Vision Research, 28(10), 1107-1118.

Morris, R. K., Rayner, K., \& Pollatsek, A. (1990). Eye movement guidance in reading: The role of parafoveal letter and space information. Journal of Experimental Psychology: Human Perception and Performance, 16(2), 268-281. doi:10.1037/ 0096-1523.16.2.268.

Morrison, R. E. (1984). Manipulation of stimulus onset delay in reading: Evidence for parallel programming of saccades. Journal of Experimental Psychology: Human Perception and Performance, 10(5), 667-682. doi:10.1037/0096-1523.10.5.667.

Muggeo, V. M. R. (2003). Estimating regression models with unknown break-points. Statistics in Medicine, 22(19), 3055-3071. doi:10.1002/sim.1545.

Muggeo, V. M. R. (2008). Segmented: An R package to fit regression models with broken-line relationships. $R$ News, 8, 20-25.

Pynte, J., \& Kennedy, A. (2006). An influence over eye movements in reading exerted from beyond the level of the word: evidence from reading English and French. Vision Research, 46(22), 3786-3801. doi:10.1016/j.visres.2006.07.004.

R Core Team. (2015). R: A language and environment for statistical computing. Vienna, Austria: R Foundation for Statistical Computing. Retrieved from https://www.r-project.org/.

Radach, R., Huestegge, L., \& Reilly, R. (2008). The role of global top-down factors in local eye-movement control in reading. Psychological Research, 72(6), 675-688. doi:10.1007/s00426008-0173-3.

Rayner, K. (1979). Eye guidance in reading: fixation locations within words. Perception, 8(1), 21-30.

Rayner, K. (1998). Eye movements in reading and information processing: 20 years of research. Psychological Bulletin, 124(3), 372-422. Retrieved from http://www.ncbi.nlm.nih.gov/pubmed/353867.

Rayner, K., Binder, K. S., Ashby, J., \& Pollatsek, A. (2001). Eye movement control in reading: word predictability has little influence on initial landing positions in words. Vision Research, 41(7), 943-954.

Rayner, K., Chace, K. H., Slattery, T. J., \& Ashby, J. (2006). Eye movements as reflections of comprehension processes in reading. Scientific Studies of Reading, 10(3), 241-255. doi:10.1207/ s1532799xssr1003_3.

Rayner, K., \& Duffy, S. A. (1986). Lexical complexity and fixation times in reading: Effects of word frequency, verb complexity, and lexical ambiguity. Memory and Cognition, 14(3), 191-201.

Rayner, K., \& Fischer, M. H. (1996). Mindless reading revisited: Eye movements during reading and scanning are different. Perception and Psychophysics, 58, 734-747.

Rayner, K., Kambe, G., \& Duffy, S. A. (2000). The effect of clause wrap-up on eye movements during reading. The Quarterly
Journal of Experimental Psychology, 53(4), 1061-1080. doi:10. 1080/713755934.

Rayner, K., \& Raney, G. E. (1996). Eye movement control in reading and visual search: Effects of word frequency. Psychonomic Bulletin \& Review, 3(2), 245-248. doi:10.3758/BF03212426.

Rayner, K., Sereno, S. C., Morris, R. K., Schmauder, A. R., \& Clifton, C. (1989). Eye movements and on-line language comprehension processes. Language and Cognitive Processes, 4(3-4), SI21SI49. doi:10.1080/01690968908406362.

Reichle, E. D., Pollatsek, A., Fisher, D. L., \& Rayner, K. (1998). Toward a model of eye movement control in reading. Psychological Review, 105(1), 125-157.

Reichle, E. D., Pollatsek, A., \& Rayner, K. (2006). E-Z Reader: A cognitive-control, serial-attention model of eye-movement behavior during reading. Cognitive Systems Research, 7(1), 4-22. doi:10.1016/j.cogsys.2005.07.002.

Reichle, E. D., Rayner, K., \& Pollatsek, A. (2003). The E-Z reader model of eye-movement control in reading: Comparisons to other models. The Behavioral and Brain Sciences, 26(4), 445-476. discussion 477-526.

Reichle, E. D., Warren, T., \& McConnell, K. (2009). Using E-Z Reader to model the effects of higher level language processing on eye movements during reading. Psychonomic Bulletin \& Review, 16(1), 1-21. doi:10.3758/PBR.16.1.1.

Schad, D. J., \& Engbert, R. (2012). The zoom lens of attention: Simulating shuffled versus normal text reading using the SWIFT model. Visual Cognition, 20(4-5), 391-421. doi:10.1080/ 13506285.2012.670143.

Schad, D. J., Nuthmann, A., \& Engbert, R. (2010). Eye movements during reading of randomly shuffled text. Vision Research, 50(23), 2600-2616. doi:10.1016/j.visres.2010.08.005.

Schmidt, R. A. (1975). A schema theory of discrete motor skill learning. Psychological Review, 82(4), 225-260. doi:10.1037/ h0076770.

Schmidt, R. A. (2003). Motor schema theory after 27 years: Reflections and implications for a new theory. Research Quarterly for Exercise and Sport, 74(4), 366-375. doi:10.1080/ 02701367.2003.10609106.

Schmidt, R. A., \& Lee, T. (2013). Motor Learning and performance, $5 E$ with web study guide: From principles to application. Champaign: Human Kinetics.

Shadmehr, R., \& Mussa-Ivaldi, F. A. (1994). Adaptive representation of dynamics during learning of a motor task. The Journal of Neuroscience, 14(5 Pt 2), 3208-3224.

Sherwood, D. E., \& Lee, T. D. (2003). Schema theory: critical review and implications for the role of cognition in a new theory of motor learning. Research Quarterly for Exercise and Sport, 74(4), 376-382. doi:10.1080/02701367.2003.10609107.

Underwood, G., Clews, S., \& Everatt, J. (1990). How do readers know where to look next? Local information distributions influence eye fixations. The Quarterly Journal of Experimental Psychology, 42(1), 39-65. doi:10.1080/14640749008401207.

Vitu, F., O'Regan, J. K., Inhoff, A. W., \& Topolski, R. (1995). Mindless reading: Eye-movement characteristics are similar in scanning letter strings and reading texts. Perception and Psychophysics, 57(3), 352-364.

Vitu, F., O'Regan, J. K., \& Mittau, M. (1990). Optimal landing position in reading isolated words and continuous text. Perception and Psychophysics, 47(6), 583-600. doi:10.3758/ BF03203111.

von der Malsburg, T., Kliegl, R., \& Vasishth, S. (2015). Determinants of scanpath regularity in reading. Cognitive Science, 39(7), 1675-1703. doi:10.1111/cogs. 12208.

von der Malsburg, T., \& Vasishth, S. (2011). What is the scanpath signature of syntactic reanalysis? Journal of Memory and Language, 65(2), 109-127. doi:10.1016/j.jml.2011.02.004. 
Warren, T., White, S. J., \& Reichle, E. D. (2009). Investigating the causes of wrap-up effects: Evidence from eye movements and E-Z Reader. Cognition, 111(1), 132-137. doi:10.1016/j.cogni tion.2008.12.011.

Williams, C. C., \& Pollatsek, A. (2007). Searching for an O in an array of Cs: eye movements track moment-to-moment processing in visual search. Perception and Psychophysics, 69(3), 372-381.
Wotschack, C. (2009). Eye movements in reading strategies: How reading strategies modulate effects of distributed processing and oculomotor control. Universität Potsdam.

Wotschack, C., \& Kliegl, R. (2013). Reading strategy modulates parafoveal-on-foveal effects in sentence reading. Quarterly Journal of Experimental Psychology, 66, 548-562. doi:10. 1080/17470218.2011.625094. 